\title{
Impact of Nursing Educational Program on Health Promotion for Patients after Intracranial Surgery
}

\author{
Rasha Ali Ahmed Abd elmowla, Zienab Abd El-Lateef Mohamad \& Roshdy Abd El-Aziz El-khayat \\ Assistant lecturer of Adult Nursing Department, Faculty of Nursing, Assiut UniversityEgypt. \\ Professor of Medical Surgical Nursing, Faculty of Nursing, Assiut University Egypt. \\ Professor of Neurosurgery, Faculty of Medicine, Assiut University Egypt.
}

\begin{abstract}
Intracranial surgery means surgery performed inside skull to treat problems in brain and surrounding structures. This study aims to: determine physical, social and emotional problems of patients after intracranial surgery, identify needs of patients after intracranial surgery and develop and implement nursing educational program based on patient's needs. Hypotheses: Studied participants patients post application of nursing educational program will exhibit more knowledge, health improvement and fewer complications. Subjects and methods: Sixty adult patients after intracranial surgery (burr hole, craniotomy and craniectomy). Their age 18-65 years old, fully conscious, both sexes. Patients divided randomly into two groups; control group (30 patients received routine care) and study group (30 patients received nursing educational program).Two tools were used for data collection; patient assessment sheet and patient evaluation sheet. Research design: Quasi- experimental research design. Results: Good improvement in total knowledge scores of study group patients after application of program. Majority of patients in study group showed health improvement while less than half of patients in control group showed health improvement. Conclusion: Improving patients` knowledge in study group had a favorable effect in improving their health than in control group. Recommendation: Establishment of educational center in neurosurgery department to educate patients about their conditions.
\end{abstract}

\section{Key words: Intracranial Surgery \& Nursing Educational Program.}

\section{Introduction}

The neurosurgical cranial procedures are relatively frequent in the daily practice and a wide range of neurosurgical techniques has been developed (burr holes, craniotomy and craniectomy) to treat patients with intracranial disorders. The entire treatment plan drains the patient physically, mentally and emotionally. It is generally the weakest and the most vulnerable phase of a patient's life (Guilabert, 2014).

Surgical burr hole is a hole made in the skull by a special drill to gain direct access of the brain (Wilson et al., 2013). A craniotomy is a surgery during which a piece of the skull (bone flap) is removed in order to allow direct access to the brain. After the surgery is performed, the bone flap is returned to its previous location. Craniectomy procedure includes removal of part of the skull bone (Heisler, 2014).

Complications after intracranial surgery may include intracranial bleeding, brain abscess, further neurological impairment, behavioral changes, infection, and seizures. Hemorrhage at the operative site can occur within hours after surgery. Bleeding may occur in the subdural or subarachnoid space or within the ventricles (Yarbro et al., 2011).

The patient needs comfort and reassurance at all times after intracranial surgery. Despite normal feelings of anxiety and fear, the caregivers should act strong and provide support with proper guidance and accurate information from the medical team. Postoperative care after intracranial surgery might not end soon in some cases; it could last for months or even for years. The caregiver should be mentally prepared for this fact and not spend all their energy right at the beginning. Also, clarify with the doctor the nature of symptoms; those which are a natural part of the recuperation process and the ones which would signal an emergency (Suzanne et al., 2010).

Because of nurses act as a vital members of the health care team and considered as a vital component in the overall patient outcome based on the expert neurological assessment, they are involved to care for intracranial surgery patient at various levels of intervention and health education. The nurse must have a thorough understanding of patients needs to provide optimal nursing intervention and education and thus improve patients health (McGlinsey \& Kirk, 2014).

Patient education is defined as any set of planned educational activities designed to improve patients' health behaviors and health status. Its main purpose is to maintain or to improve patient health or, in some cases, to slow deterioration. An informed and educated patient can actively participate in his or her own treatment, improve outcomes, help identify 
errors before they occur, and reduce his or her length of stay. Medical component of health education involves medical information and preventative measures concerning health and well-being. Research has demonstrated that effective health education begins with identification of various important needs for the patients (Haddad et al., 2013). Health promotion is the science and art of helping people change their lifestyle to move toward a state of optimal health. Optimal health is defined as a balance of physical, emotional, social, spiritual and intellectual health. Lifestyle change can be facilitated through a combination of efforts to enhance awareness, change behavior and create environments that support good health practices (World Health Organization, 2012).

According to hospital records at Assiut University Hospital during the period of one year (1/1/2013$1 / 1 / 2014)$, it has been found that 998 patients admitted to the neurosurgery department and perform intracranial surgery (Assiut University Hospital Record, 2013-2014). Not present international incidence related to intracranial surgery but there is incidence for the diseases that require intracranial surgery.

\section{Significance of the study}

Patients after intracranial surgery have physical, social, and emotional problems that affect greatly their life. Those patients are needed for special nursing care and health teaching to improve their condition. So, this study will be the first study in this geographical location which will help those patients improve their condition

\section{Aim of the study}

\section{This study aimed to:}

1. Determine the physical, social and emotional problems of patients after intracranial surgery.

2. Identify the needs of patients after intracranial surgery.

3. Develop and implement the nursing educational program based on patient's needs.

\section{Research hypotheses}

To fulfill the aim of this study, the following research hypotheses were formulated:

1. The knowledge of studied patients after application of the nursing educational program will be higher than their knowledge before the application of it.

2. Studied participants patients post application of nursing educational program will exhibit higher health improvement than control group.

3. Studied participants patients post application of nursing educational program will exhibit fewer symptoms or complications; physical, social or emotional problems than control group.

\section{Subjects and methods}

\section{Research design:}

Quasi experimental research design was utilized to fulfill the aims of this study.

\section{Technical design}

Setting

This study was conducted in neurosurgery department and neurosurgery out patient clinic at Assiut University Hospital.

\section{Subjects}

A random sample was obtained; sixty adult patients after intracranial surgery (burr hole, craniotomy and craniectomy) were included in this study. They had the following criteria; the age ranged from18-65 years old, fully conscious, both males and females. The patients divided randomly into two groups: the control group (30 patients who received routine care) and the study group (30 patients who received nursing educational program). The number of males include: (18 in control and 17 in study groups) while the number of females include: (12 in control and 13 in study groups). Data were collected during the period from (December 2012 to June 2014); this period included both the time of assessment and follow up. Patients were assessed immediately after intracranial surgery (burr hole, craniotomy and craniectomy) and daily during hospitalization. Those patients were followed up before discharge in the neurosurgery department, 3 months and 6 months after intracranial surgery in the neurosurgery out patient clinic. Their ages ranged from 18 to 65 years with a mean value of $(43.27 \pm 6.38)$ for control and (41.25 \pm 5.83$)$ for study groups.

\section{Exclusion criteria}

- Disoriented patient.

- Uncooperative patient.

- Patients on mechanical ventilation.

Tools

Tool I: Postoperative intracranial surgery patient assessment sheet: This tool consisted of four parts:

Part 1: Socio-demographic characteristics of patients.

Part 2: Patients' nursing needs: This included structured items to identify patients`nursing needs; it is used to assess:

1. Medical history.

2. Neurological physical examination (level of consciousness by Glasgow coma scale (GCS) (Teasdale and Jennett, 1974), cranial nerves, motor and sensory function, sphincter control and vital signs). 
3. Psychological problems (social and emotional problems) such as anxiety, fear, depression or social isolation, sensitivity to noise or people in crowded places, apathy or crying spells.

4. Laboratory investigation and diagnostic procedures. It includes certain investigation such as hemoglobin level, red and white blood cells count, platelets count, blood sugar level, kidney function (urea and creatinine), prothrombin time and concentration and/or serum electrolytes (sodium, potassium). Certain procedures such as computed tomography, magnetic resonance imaging and cerebrospinal fluid analysis.

Part 3: Systemic and/or neurosurgical postoperative complications that may develop for patients after intracranial surgery. Systemic complications (cardiovascular, gastrointestinal, metabolic and wound infection). Neurosurgical complications (behavioral changes, cerebrospinal fluid leak, postoperative hematoma, seizures, residual neurological problems, visual disturbances brain abscess and insomnia)

Part 4: Patients' knowledge regarding intracranial surgery: It included 39 questions concerning types of intracranial surgery, treatment, postoperative complications, postoperative care, follow up and health promotion after intracranial surgery.

Nursing educational program after intracranial surgery: Developed by researcher based on patient's assessment needs after reviewing current national and international literature to maintain health promotion for patients and reduce or prevent postoperative complications. It includes:

- Brief anatomy of the brain.

- Definition and indication for intracranial surgery.

- Various diagnostic procedures and how to be prepared for it.

- Benefits of surgical management and types of intracranial surgery.

- Postoperative complications related to anesthesia and surgery.

- Physical, social and emotional problems that the patient may complain after intracranial surgery.
- Information about how to promote health through:

- Medical therapy after intracranial surgery.

- How to deal with seizures.

- Care of wound site.

- Routine follow up and when it is necessary to seek medical help and immediately go to the hospital

- Life style modification:

- Nutrition.

- Weight control.

- Rest.

- Physical activity and exercises.

- Smoking cessation.

- Stress reduction.

- Effective communication.

- Control of diabetes mellitus and hypertension.

Tool II: Patient evaluation sheet: This tool consisted of three parts:

Part 1: Patients' knowledge regarding intracranial surgery, it included 39 questions concerning types of intracranial surgery, treatment, postoperative complications, postoperative care, follow up and health promotion after intracranial surgery.

Part 2: Rand short form (SF) 36 items questionnaire. It contains 36 questions and measures health status. It divided into eight scales plus one health comparison question (health change).

1. Physical functioning.

2. Role limitations due to physical problems.

3. Role limitations due to emotional problems.

4. Vitality (Energy / fatigue).

5. Mental health (Emotional well being).

6. Social functioning.

7. Pain.

8. General health.

- Health comparison question (Health change).

Scoring system for Rand short form 36 questionnaire (Ware and Sherbourne, 1992): Each scale contains from 2-10 items. All questions are scored on a scale from 0 to 100 , with 100 representing the highest level.

\section{How to score Rand short form-36 questionnaire} Step 1: Scoring questions

\begin{tabular}{|l|c|c|}
\hline \multicolumn{2}{|c|}{ Scoring system } \\
\hline Question number & Original response & Recorded value \\
\hline $1,2,20,22,34,36$ & 1 & 100 \\
\cline { 2 - 3 } & 2 & 75 \\
\cline { 2 - 3 } & 3 & 50 \\
\cline { 2 - 3 } & 4 & 0 \\
\hline
\end{tabular}




\begin{tabular}{|c|c|c|}
\hline \multicolumn{3}{|c|}{ Scoring system } \\
\hline Question number & Original response & Recorded value \\
\hline \multirow[t]{3}{*}{$3,4,5,6,7,8,9,10,11,12$} & 1 & 0 \\
\hline & 2 & 50 \\
\hline & 3 & 100 \\
\hline \multicolumn{3}{|c|}{ Scoring system } \\
\hline Question number & Original response & Recorded value \\
\hline \multirow[t]{2}{*}{$13,14,15,16,17,18,19$} & 1 & 0 \\
\hline & 2 & 100 \\
\hline \multicolumn{3}{|c|}{ Scoring system } \\
\hline Question number & Original response & Recorded value \\
\hline \multirow[t]{5}{*}{21} & 1 & 100 \\
\hline & 2 & 80 \\
\hline & 3 & 60 \\
\hline & 4 & 40 \\
\hline & 5 & 20 \\
\hline \multicolumn{3}{|c|}{ Scoring system } \\
\hline Question number & Original response & Recorded value \\
\hline \multirow[t]{6}{*}{$23,26,27,30$} & 1 & 100 \\
\hline & 2 & 80 \\
\hline & 3 & 60 \\
\hline & 4 & 40 \\
\hline & 5 & 20 \\
\hline & 6 & 0 \\
\hline \multicolumn{3}{|c|}{ Scoring system } \\
\hline Question number & Original response & Recorded value \\
\hline \multirow[t]{6}{*}{$24,25,28,29,31$} & 1 & 0 \\
\hline & 2 & 20 \\
\hline & 3 & 40 \\
\hline & 4 & 60 \\
\hline & 5 & 80 \\
\hline & 6 & 100 \\
\hline \multicolumn{3}{|c|}{ Scoring system } \\
\hline Question number & Original response & Recorded value \\
\hline \multirow[t]{5}{*}{$32,33,35$} & 1 & 0 \\
\hline & 2 & 25 \\
\hline & 3 & 50 \\
\hline & 4 & 75 \\
\hline & 5 & 100 \\
\hline
\end{tabular}

Step 2: Average items to form 8 scales

\begin{tabular}{|l|c|c|}
\hline \multicolumn{1}{|c|}{ Scale } & Number of items & $\begin{array}{c}\text { Average the following items } \\
\text { (Question numbers) }\end{array}$ \\
\hline $\begin{array}{l}\text { Physical functioning; Limitations in physical activity } \\
\text { because of health problems }\end{array}$ & 10 & $3,4,5,6,7,8,9,10,11,12$ \\
\hline $\begin{array}{l}\text { Role limitations - physical; Limitations in usual role } \\
\text { activities because of physical health problem }\end{array}$ & 4 & $13,14,15,16$ \\
\hline $\begin{array}{l}\text { Role limitations - emotional; Limitations in usual } \\
\text { role activities because of emotional problems. }\end{array}$ & 3 & $17,18,19$ \\
\hline Vitality; Energy and fatigue & 4 & $23,27,29,31$ \\
\hline
\end{tabular}




\begin{tabular}{|l|c|c|}
\hline \multicolumn{1}{|c|}{ Scale } & Number of items & $\begin{array}{c}\text { Average the following items } \\
\text { (Question numbers) }\end{array}$ \\
\hline Mental health; Psychological distress and well-being. & 5 & $24,25,26,28,30$ \\
\hline $\begin{array}{l}\text { Social functioning; Limitations in social activities } \\
\text { because of physical or emotional problems }\end{array}$ & 2 & 20,32 \\
\hline Pain; Presence of pain and limitations due to pain & 2 & 21,22 \\
\hline General health perception & 5 & $1,33,34,35,36$ \\
\hline Health comparison question; Health change & 1 & 2 \\
\hline
\end{tabular}

\section{Step 3: Figuring scores}

Rand recommends the following straightforward approach to scoring the Rand 36-items health survey. All questions are scored on a scale from 0 to 100 , with 100 representing the highest level.

Aggregate scores are compiled as a percentage of the total points possible, using the Rand scoring table (step 1).

The scores from those questions that address each specific area of functional health status (step 2) are then averaged together, for a final score within each of the 8 scales measured (e.g. pain, physical functioning etc.)

For example, to measure the patient's energy/fatigue level, add the scores from questions 23, 27, 29, and 31. If a patient circled 4 on 23, 3 on 27, 3 on 29 and 1 on 31 , use (Step 1) to score them.

An answer of 4 to question 23 is scored as 40,3 to question 27 is scored as 60,3 to question 29 is scored as 40 and 1 to question 31 is scored as 0 . The score for this block is $40+60+40+0=140$. Now we divide by the 4 answered questions to get a total of 46.7. Since a score of 100 represents high energy with no fatigue, the lower score of $46.7 \%$ suggests the patient is experiencing a loss of energy and is experiencing some fatigue. All 8 scales are scored in the same way. Part 3: Systemic and/or neurosurgical postoperative complications that may develop for patients after intracranial surgery. Systemic complications (cardiovascular, gastrointestinal, metabolic and wound infection). Neurosurgical complications (behavioral changes, cerebrospinal fluid leak, postoperative hematoma, seizures, residual neurological problems, visual disturbances brain abscess and insomnia)

\section{Operational design}

Procedure:

Phase I: Preparatory phase: An official permission to proceed with the proposed study was obtained from the head of the neurosurgery department. The researcher designed and tested health promotion program after reviewing extensive literature.

\section{Content validity and reliability}

Content validity was done by five expertise from the medical staff and medical -surgical nursing staff. Two expertise from medical-surgical nursing staff and three expertise from neurosurgery staff who reviewed the tools and the nursing educational program for clarity, relevance, comprehensiveness, understanding, applicability and easiness for administration. The content is valid and reliable. Reliability was assessed by correlation coefficient with $>0.8$ considered strong.

Pilot study:

It was conducted on $10 \%$ of sample (6 patients) in a selected setting for testing clarity and applicability and feasibility of the study tools. The purpose of the pilot study was to detect any particular problem in the statements clarity, feasibility, and applicability of the tool. The data obtained from the pilot study were analyzed, no change was done in the assessment sheet, so the $10 \%$ of subjects selected for the pilot study were included in the main study.

Phase II: implementation phase: At initial interview the researcher introduce herself to initiate communication, explain the nature and purpose of the study.

The study group visited daily during hospitalization to fill out the patient assessment sheet (tool I) to assess patients' knowledge and needs and implement nursing educational program.

Regarding control group, they received the routine postoperative hospital care. They visited daily during hospitalization to fill out (tool I).

The nursing educational program was introduced to patients through individualized sessions. 10 educational sessions were conducted for each patient. The duration of each session was $30-45$ minutes, including 10 minutes for discussion and feedback. Each session usually started by a summary of what has been taught during the previous sessions and the objectives of the new topics. Feedback and reinforcement of teaching was performed according to the patients` needs to ensure their understanding. Each session ended by a summary of its contents and feedback of the patient through discussion and questions.

Phase III: Evaluation phase: After application of the nursing educational program, the patients' knowledge and condition have been evaluated by the researcher through filling the patient evaluation sheet (tool II). Follow up has been done for patients in 
study and control groups for a period of 6 months on three phases (before discharge, 3months and 6 months after intracranial surgery).

Ethical considerations

Informed consent was obtained from patients who are willing to participate in the study after explanation of the nature and purposes of the study. Patients privacy and confidentiality were certainly assured.

\section{Statistical design}

Data were collected and analyzed by computer program SPSS (version 17). Data expressed as mean, standard deviation, number and percentage. T-test is used to determine significant for numeric variable. Chi-square test is used to determine significant for non-parametric variable.

A probability level of $<0.05$ was adopted as a level of significance for testing the research hypotheses.

Limitations of the study

1. The patient's anxiety may interfere with the ability to learn information provided.

2. During preparation of a teaching booklet, the level of literacy in the patient population was considered. A lack of reading skills limited the ability of patients to access and use critical information.

3. Participants suffered from transportation and financial problems. So, some patients interview after 6 months by telephone.

\section{Results}

Table (1): Distribution of socio-demographic characteristics of patients (control and study groups).

\begin{tabular}{|c|c|c|c|c|c|c|}
\hline \multirow{2}{*}{$\begin{array}{c}\text { Socio-demographic } \\
\text { characteristics }\end{array}$} & \multicolumn{3}{|c|}{ Control $(n=30)$} & \multicolumn{3}{|c|}{ Study $(n=30)$} \\
\hline & No. & $\%$ & $\mathrm{X}+\mathrm{SD}$ & No. & $\%$ & $\mathrm{X}+\mathrm{SD}$ \\
\hline $\begin{array}{l}\text { Age } \\
18<35 \text { yrs } \\
35<50 y r s \\
50-65 y r s\end{array}$ & $\begin{array}{c}1 \\
19 \\
10\end{array}$ & $\begin{array}{c}3.3 \\
63.3 \\
33.3 \\
\end{array}$ & $43.27 \pm 6.38$ & $\begin{array}{c}3 \\
17 \\
10\end{array}$ & $\begin{array}{c}10 \\
56.7 \\
33.3\end{array}$ & $41.25 \pm 5.83$ \\
\hline $\begin{array}{l}\text { Sex } \\
\text { Male } \\
\text { Female }\end{array}$ & $\begin{array}{l}18 \\
12\end{array}$ & $\begin{array}{l}60 \\
40\end{array}$ & - & $\begin{array}{l}17 \\
13\end{array}$ & $\begin{array}{l}56.7 \\
43.3\end{array}$ & - \\
\hline $\begin{array}{l}\text { Marital status } \\
\text { Single } \\
\text { Married }\end{array}$ & $\begin{array}{l}11 \\
19\end{array}$ & $\begin{array}{l}36.7 \\
63.3\end{array}$ & - & $\begin{array}{c}4 \\
26\end{array}$ & $\begin{array}{l}13.3 \\
86.7\end{array}$ & - \\
\hline $\begin{array}{l}\text { Level of education } \\
\text { High education } \\
\text { Secondary school education } \\
\text { Read and write } \\
\text { Illiterate }\end{array}$ & $\begin{array}{c}2 \\
6 \\
5 \\
17\end{array}$ & $\begin{array}{c}6.7 \\
20 \\
16.7 \\
56.7\end{array}$ & - & $\begin{array}{c}2 \\
8 \\
6 \\
14\end{array}$ & $\begin{array}{c}6.7 \\
26.7 \\
20 \\
46.7\end{array}$ & - \\
\hline $\begin{array}{l}\text { Occupation } \\
\text { Office work } \\
\text { Machinery work } \\
\text { Manual work } \\
\text { Housewife } \\
\text { Not working }\end{array}$ & $\begin{array}{c}3 \\
6 \\
14 \\
6 \\
1\end{array}$ & $\begin{array}{c}10 \\
20 \\
46.7 \\
20 \\
3.3\end{array}$ & - & $\begin{array}{c}5 \\
2 \\
11 \\
9 \\
3\end{array}$ & $\begin{array}{c}16.7 \\
6.7 \\
36.7 \\
30.0 \\
10\end{array}$ & - \\
\hline
\end{tabular}

Table (2): Distribution of patients`medical history (control and study groups).

\begin{tabular}{|c|c|c|c|c|}
\hline \multirow{2}{*}{ Patients` medical history } & \multicolumn{2}{|c|}{ Control $(\mathrm{n}=30)$} & \multicolumn{2}{|c|}{ Study $(n=30)$} \\
\hline & No. & $\%$ & No. & $\%$ \\
\hline \multicolumn{5}{|l|}{ Surgical management } \\
\hline Burr holes & 8 & 26.7 & 12 & 40.0 \\
\hline Craniotomy & 18 & 60.0 & 13 & 43.33 \\
\hline Craniectomy & 4 & 13.33 & 5 & 16.7 \\
\hline
\end{tabular}


Table (3): Distribution of patients (study and control groups) as regard level of consciousness (GCS).

\begin{tabular}{|c|c|c|c|c|c|c|}
\hline \multirow{2}{*}{ GCS } & \multicolumn{3}{|c|}{ Control (n =30) } & \multicolumn{3}{|c|}{ Study $(n=30)$} \\
\hline & No. & $\%$ & $\mathrm{X} \pm \mathrm{SD}$ & No. & $\%$ & $\mathrm{X} \pm \mathrm{SD}$ \\
\hline Mild & 30 & 100 & \multirow{3}{*}{$15.00 \pm 15.00$} & 30 & 100 & \multirow[t]{3}{*}{$15.00 \pm 15.00$} \\
\hline Moderate & - & - & & - & - & \\
\hline Severe & - & - & & - & - & \\
\hline
\end{tabular}

Table (4): Distribution of patients (study and control groups) as regard cranial nerves.

\begin{tabular}{|c|c|c|c|c|c|c|c|c|c|}
\hline \multirow{3}{*}{ Cranial nerves } & \multicolumn{4}{|c|}{ Control $(\mathbf{n}=\mathbf{3 0})$} & \multicolumn{4}{|c|}{ Study $(n=30)$} & \multirow[t]{3}{*}{ P-value } \\
\hline & \multicolumn{2}{|c|}{ Normal } & \multicolumn{2}{|c|}{ Abnormal } & \multicolumn{2}{|c|}{ Normal } & \multicolumn{2}{|c|}{ Abnormal } & \\
\hline & No. & $\%$ & No. & $\%$ & No. & $\%$ & No. & $\%$ & \\
\hline I- Olfactory & 30 & 100 & - & - & 30 & 100 & - & - & \\
\hline II- Optic & 27 & 90 & 3 & 10 & 28 & 93.3 & 2 & 6.7 & \\
\hline III- Oculomotor & 30 & 100 & - & - & 30 & 100 & - & - & $\mathrm{P}=0.694$ \\
\hline IV- Trochlear & 30 & 100 & - & - & 30 & 100 & - & - & $\mathrm{n} . \mathrm{s}$ \\
\hline V- Trigeminal & 30 & 100 & - & - & 30 & 100 & - & - & \\
\hline VI- Abducens & 30 & 100 & - & - & 30 & 100 & - & - & \\
\hline VII- Facial & 30 & 100 & - & - & 30 & 100 & - & - & \\
\hline VIII- Acoustic & 29 & 96.7 & 1 & 3.3 & 30 & 100 & - & - & \\
\hline IX- Glosopharyngeal & 30 & 100 & - & - & 30 & 100 & - & - & \\
\hline $\mathrm{X}$ - Vagus & 30 & 100 & - & - & 30 & 100 & - & - & \\
\hline XI- Accessory & 30 & 100 & - & - & 30 & 100 & - & - & \\
\hline XII- Hypoglossal & 30 & 100 & - & - & 30 & 100 & - & - & \\
\hline
\end{tabular}

Table (5): Distribution of patients (study and control groups) as regard sensory function.

\begin{tabular}{|c|c|c|c|c|c|c|c|c|c|}
\hline \multirow{3}{*}{ Sensory function } & \multicolumn{4}{|c|}{ Control $(\mathbf{n}=\mathbf{3 0})$} & \multicolumn{4}{|c|}{ Study $(\mathrm{n}=30)$} & \multirow{2}{*}{ P-value } \\
\hline & \multicolumn{2}{|c|}{ Normal } & \multicolumn{2}{|c|}{ Abnormal } & \multicolumn{2}{|c|}{ Normal } & \multicolumn{2}{|c|}{ Abnormal } & \\
\hline & No. & $\%$ & No. & $\%$ & No. & $\%$ & No. & $\%$ & \multirow{4}{*}{$\mathrm{P}=0.762 \mathrm{n} . \mathrm{s}$} \\
\hline Pain and temperature sensation & 30 & 100 & - & - & 30 & 100 & 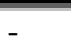 & 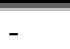 & \\
\hline Position sense (Proprioception) & 29 & 96.7 & 1 & 3.3 & 30 & 100 & - & - & \\
\hline Light touch & 28 & 93.3 & 2 & 6.7 & 29 & 96.7 & 1 & 3.3 & \\
\hline
\end{tabular}

Table (6): Distribution of patients (study and control groups) as regard sphincter control.

\begin{tabular}{|c|c|c|c|c|c|c|c|c|c|}
\hline \multirow{3}{*}{ Sphincter control } & \multicolumn{4}{|c|}{ Control $(\mathrm{n}=30)$} & \multicolumn{4}{|c|}{ Study $(\mathrm{n}=\mathbf{3 0})$} & \multirow[t]{2}{*}{ P-value } \\
\hline & \multicolumn{2}{|c|}{ Present } & \multicolumn{2}{|c|}{ Absent } & \multicolumn{2}{|c|}{ Present } & \multicolumn{2}{|c|}{ Absent } & \\
\hline & No. & $\%$ & No. & $\%$ & No. & $\%$ & No. & $\%$ & \\
\hline Bladder control & 29 & 96.7 & 1 & 3.3 & 30 & 100 & - & - & \\
\hline Bowel control & 30 & 100 & - & - & 30 & 100 & - & - & $0.794 n . s$ \\
\hline
\end{tabular}


Table (7): Distribution of patients (study and control groups) as regard motor function.

\begin{tabular}{|c|c|c|c|c|c|}
\hline \multirow{2}{*}{ Motor function } & \multicolumn{2}{|c|}{ Control $(\mathrm{n}=30)$} & \multicolumn{2}{|c|}{ Study $(\mathrm{n}=30)$} & \multirow{2}{*}{ P-value } \\
\hline & No. & $\%$ & No. & $\%$ & \\
\hline \multicolumn{5}{|l|}{ 1. Muscle size } & \multirow{4}{*}{-} \\
\hline Normal & 30 & 100 & 30 & 100 & \\
\hline Muscle atrophy & - & - & - & - & \\
\hline \multicolumn{5}{|l|}{ 2. Muscle tone } & \\
\hline Normal & 30 & 100 & 30 & 100 & \multirow{4}{*}{-} \\
\hline Spastic & - & - & - & - & \\
\hline Rigid & - & - & - & - & \\
\hline Flaccid & - & - & - & - & \\
\hline \multicolumn{5}{|l|}{ 3. Muscle power } & \multirow{7}{*}{$P=0.859 n . s$} \\
\hline Grade 5: Normal power & 28 & 93.3 & 30 & 100 & \\
\hline $\begin{array}{l}\text { Grade 4: Active movement against gravity and } \\
\text { resistance }\end{array}$ & 2 & 6.7 & - & - & \\
\hline Grade 3: Active movement against gravity & - & - & - & - & \\
\hline Grade 2: Active movement with gravity eliminated & - & - & - & - & \\
\hline Grade 1: Trace of contraction & - & - & - & - & \\
\hline Grade 0: Absent, no muscle contraction & - & - & - & - & \\
\hline \multicolumn{5}{|l|}{ 5. Involuntary movements } & \multirow{3}{*}{$\mathrm{P}=0.793 \mathrm{n} . \mathrm{s}$} \\
\hline Yes & 2 & 6.7 & 1 & 3.3 & \\
\hline No & 28 & 93.3 & 29 & 96.7 & \\
\hline
\end{tabular}

Table (8): Distribution of patients (study and control groups) as regard vital signs.

\begin{tabular}{|l|c|c|c|}
\hline \multicolumn{1}{|c|}{ Vital signs } & $\begin{array}{c}\text { Control }(\mathbf{n}=\mathbf{3 0}) \\
\mathbf{X} \pm \text { SD }\end{array}$ & $\begin{array}{c}\text { Study }(\mathbf{n}=\mathbf{3 0}) \\
\mathbf{X} \pm \text { SD }\end{array}$ & P-value \\
\hline Body temperature & & & \\
1 st day & $36.86 \pm 0.13$ & $36.83 \pm 0.19$ & $\mathrm{P}=0.531 \mathrm{n} . \mathrm{s}$ \\
2 nd day & $36.94 \pm 0.08$ & $36.93 \pm 0.15$ & $\mathrm{P}=0.425 \mathrm{n} . \mathrm{s}$ \\
3 rd day & $37.85 \pm 0.39$ & $36.92 \pm 0.15$ & $\mathrm{P}<0.01^{*}$ \\
\hline Pulse & & & \\
1 st day & $76.53 \pm 12.00$ & $78.63 \pm 6.50$ & $\mathrm{P}=0.429 \mathrm{n} . \mathrm{s}$ \\
2 nd day & $78.83 \pm 11.91$ & $80.50 \pm 5.32$ & $\mathrm{P}=0.372 \mathrm{n} . \mathrm{s}$ \\
3 rd day & $77.00 \pm 11.84$ & $79.80 \pm 4.55$ & $\mathrm{P}<0.01^{*}$ \\
\hline Respiration: & & & \\
1 st day & $18.16 \pm 1.17$ & $18.63 \pm 1.86$ & $\mathrm{P}=0.441 \mathrm{n} . \mathrm{s}$ \\
2 nd day & $18.83 \pm 1.46$ & $18.56 \pm 1.77$ & $\mathrm{P}=0.588 \mathrm{n} . \mathrm{s}$ \\
3 rd day & $18.93 \pm 1.38$ & $18.20 \pm 1.39$ & $\mathrm{P}<0.04 *$ \\
\hline Blood pressure & Systolic & Systolic & \\
1 st day & $116.50 \pm 8.00$ & $121.17 \pm 9.79$ & $\mathrm{P}=0.522 \mathrm{n} . \mathrm{s}$ \\
2 nd day & $117.17 \pm 5.97$ & $116.40 \pm 20.25$ & $\mathrm{P}=0.621 \mathrm{n} . \mathrm{s}$ \\
3 rd day & $116.67 \pm 5.77$ & $115.57 \pm 19.66$ & $\mathrm{P}<0.04 *$ \\
\cline { 2 - 4 } 1 st day & Diastolic & Diastolic & \multirow{2}{*}{ P $=0.502 \mathrm{n} . \mathrm{s}$} \\
2 nd day & $80.16 \pm 8.55$ & $81.50 \pm 6.58$ & $\mathrm{P}=0.264$ n.s \\
3 rd day & $80.03 \pm 4.77$ & $81.50 \pm 5.27$ & $\mathrm{P}<0.02^{*}$ \\
\hline
\end{tabular}


Table (9): Distribution of patients (study and control groups) as regard psychological assessment (social and emotional problems).

\begin{tabular}{|l|c|c|c|c|c|}
\hline \multicolumn{1}{|c|}{$\begin{array}{c}\text { Psychological assessment } \\
\text { (More than one) }\end{array}$} & \multicolumn{2}{c|}{ Control (n=30) } & \multicolumn{2}{c|}{ Study (n=30) } & \multirow{2}{*}{ P-value } \\
\cline { 2 - 5 } & No. & \% & No. & \% & P =0.246 n.s \\
\hline 1. Anxiety & 28 & 93.3 & 27 & 90.0 & $\mathrm{P}=0.335$ n.s \\
\hline 2. Fear & 28 & 93.3 & 27 & 90.0 & $\mathrm{P}=0.246$ n.s \\
\hline 3. Depression (social isolation) & 2 & 6.7 & - & - & $\mathrm{P}=0.212$ n.s \\
\hline 4. Sensitivity to noise & 5 & 16.7 & 2 & 6.7 & \\
\hline
\end{tabular}

Table (10): Comparison between patients (study and control groups) as regard laboratory investigation.

\begin{tabular}{|l|c|c|c|}
\hline \multicolumn{1}{|c|}{ Laboratory investigation } & Control $(\mathbf{n}=\mathbf{3 0}) \mathbf{X} \pm$ SD & Study $(\mathbf{n}=30) \mathbf{X} \pm$ SD & P-value \\
\hline \hline Hemoglobin & $13.64 \pm 1.26$ & $13.66 \pm 1.05$ & $\mathrm{P}=0.962 \mathrm{n} . \mathrm{s}$ \\
\hline White blood cells & $7.08 \pm 2.75$ & $6.92 \pm 2.11$ & $\mathrm{P}=0.791 \mathrm{n} . \mathrm{s}$ \\
\hline Red blood cells & $5.04 \pm 0.506$ & $4.85 \pm 0.52$ & $\mathrm{P}=0.151 \mathrm{n} . \mathrm{s}$ \\
\hline Platelets & $239.33 \pm 53.32$ & $248.53 \pm 55.70$ & $\mathrm{P}=0.516 \mathrm{n} . \mathrm{s}$ \\
\hline Blood sugar & $5.94 \pm 2.25$ & $6.31 \pm 1.21$ & $\mathrm{P}=0.432 \mathrm{n} . \mathrm{s}$ \\
\hline Urea & $5.27 \pm 1.54$ & $5.43 \pm 1.25$ & $\mathrm{P}=0.667 \mathrm{n} . \mathrm{s}$ \\
\hline Creatinine & $81.08 \pm 17.75$ & $85.34 \pm 16.93$ & $\mathrm{P}=0.346 \mathrm{n} . \mathrm{s}$ \\
\hline Prothrombin time & $16.20 \pm 2.48$ & $12.06 \pm 0.46$ & $\mathrm{P}=0.317 \mathrm{n} . \mathrm{s}$ \\
\hline Prothrombin concentration & $94.07 \pm 18.33$ & $97.40 \pm 5.22$ & $\mathrm{P}=0.343 \mathrm{n} . \mathrm{s}$ \\
\hline Sodium & $144.52 \pm 4.11$ & $139.97 \pm 3.95$ & $\mathrm{P}=0.117 \mathrm{n} . \mathrm{s}$ \\
\hline Potassium & $4.23 \pm 0.32$ & $4.04 \pm 0.43$ & $\mathrm{P}=0.489 \mathrm{n} . \mathrm{s}$ \\
\hline
\end{tabular}

Table (11): Comparison between patients (study and control groups) as regard diagnostic procedure.

\begin{tabular}{|l|c|c|c|c|c|}
\hline \multicolumn{1}{|c|}{$\begin{array}{c}\text { Diagnostic procedure } \\
\text { (More than one) }\end{array}$} & \multicolumn{2}{c|}{ Control (n=30) } & \multicolumn{2}{c|}{ Study (n =30) } & \multirow{2}{*}{ P-value } \\
\cline { 2 - 6 } & No. & $\mathbf{\%}$ & No. & \% & P=0.35 n.s \\
\hline Computed tomography & 3 & 10 & 5 & 16.7 & $\mathrm{P}=0.50 \mathrm{n} . \mathrm{s}$ \\
\hline Magnetic resonance imaging & 2 & 6.7 & 1 & 3.3 & $\mathrm{P}=0.50 \mathrm{n} . \mathrm{s}$ \\
\hline Cerebrospinal fluid analysis & 1 & 3.3 & - & - & - \\
\hline Electroencephalogram & - & - & - & - & - \\
\hline X-rays & - & - & - & - & - \\
\hline Others & - & - & - & - & - \\
\hline
\end{tabular}

Table (12): Distribution of patients (study and control groups) as regard systemic postoperative complications at assessment.

\begin{tabular}{|c|c|c|c|c|c|}
\hline \multirow{2}{*}{$\begin{array}{l}\text { Systemic postoperative complications } \\
\text { (More than one) }\end{array}$} & \multicolumn{2}{|c|}{ Control $(\mathbf{n}=30)$} & \multicolumn{2}{|c|}{ Study $(n=30)$} & \multirow[b]{2}{*}{ P-value } \\
\hline & No. & $\%$ & No. & $\%$ & \\
\hline $\begin{array}{l}\text { 1. Cardiovasular complications } \\
\text { - Hypotension } \\
\text { - Hypertension } \\
\text { - Bradycardia } \\
\text { - Tachycardia } \\
\text { - Myocardial infarction } \\
\text { - Arrhythmias } \\
\text { - Heart failure }\end{array}$ & $\begin{array}{l}- \\
5 \\
- \\
5 \\
- \\
- \\
-\end{array}$ & $\begin{array}{c}- \\
16.7 \\
- \\
16.7 \\
- \\
- \\
-\end{array}$ & $\begin{array}{l}- \\
2 \\
- \\
2 \\
- \\
- \\
-\end{array}$ & $\begin{array}{l}- \\
6.7 \\
- \\
6.7 \\
- \\
- \\
-\end{array}$ & \multirow[t]{2}{*}{$\begin{array}{c}\mathrm{P}=0.273 \\
\text { n.s }\end{array}$} \\
\hline $\begin{array}{l}\text { 2. Gastrointestinal complications } \\
\text { - Nausea } \\
\text { - Vomiting } \\
\text { - Gastric irritation } \\
\text { - Gastric stress ulceration } \\
\text { - Hemorrhage }\end{array}$ & $\begin{array}{l}2 \\
2 \\
- \\
- \\
-\end{array}$ & $\begin{array}{c}6.7 \\
6.7 \\
- \\
- \\
-\end{array}$ & $\begin{array}{l}4 \\
4 \\
- \\
- \\
-\end{array}$ & $\begin{array}{c}13.3 \\
13.3 \\
- \\
- \\
-\end{array}$ & \\
\hline
\end{tabular}


Table (13): Distribution of patients (study and control groups) as regard neurosurgical postoperative complications at assessment.

\begin{tabular}{|c|c|c|c|c|c|}
\hline \multirow{2}{*}{$\begin{array}{c}\text { Neurosurgical postoperative complications } \\
\text { (More than one) }\end{array}$} & \multicolumn{2}{|c|}{ Control $(n=30)$} & \multicolumn{2}{|c|}{ Study $(n=30)$} & \multirow[t]{2}{*}{ P-value } \\
\hline & No. & $\%$ & No. & $\%$ & \\
\hline 1. Cerebral edema & - & - & - & - & \multirow{9}{*}{$\begin{array}{c}\mathrm{P}=0.338 \\
\text { n.s }\end{array}$} \\
\hline 2. Brain or nerve damage & - & - & - & - & \\
\hline 3.Stroke & - & - & - & - & \\
\hline 4. Behavioral changes & 2 & 6.7 & - & - & \\
\hline 5. Cerebrospinal fluid leak & 1 & 3.3 & 1 & 3.3 & \\
\hline 6. Postoperative subdural hematoma & 2 & 6.7 & - & - & \\
\hline 7. Pneumocephalus & - & - & - & - & \\
\hline 8. Seizures & 3 & 10.0 & 2 & 6.7 & \\
\hline 9. Others & - & - & - & - & \\
\hline
\end{tabular}

Table (14): Comparison between systemic postoperative complications of patients (study and control groups) at follow up.

\begin{tabular}{|c|c|c|c|c|c|c|c|c|c|c|c|c|}
\hline \multirow{3}{*}{$\begin{array}{c}\text { Systemic } \\
\text { postoperative } \\
\text { complications } \\
\text { (More than one) }\end{array}$} & \multicolumn{4}{|c|}{ Before discharge } & \multicolumn{4}{|c|}{ After 3 months } & \multicolumn{4}{|c|}{ After 6 months } \\
\hline & \multicolumn{2}{|c|}{$\begin{array}{l}\text { Control } \\
(\mathbf{n}=\mathbf{3 0})\end{array}$} & \multicolumn{2}{|c|}{$\begin{array}{c}\text { Study } \\
(\mathbf{n}=\mathbf{3 0})\end{array}$} & \multicolumn{2}{|c|}{$\begin{array}{l}\text { Control } \\
(\mathbf{n}=\mathbf{3 0})\end{array}$} & \multicolumn{2}{|c|}{$\begin{array}{c}\text { Study } \\
(\mathbf{n}=\mathbf{3 0})\end{array}$} & \multicolumn{2}{|c|}{$\begin{array}{l}\text { Control } \\
(\mathbf{n}=\mathbf{3 0})\end{array}$} & \multicolumn{2}{|c|}{$\begin{array}{c}\text { Study } \\
(\mathbf{n}=\mathbf{3 0})\end{array}$} \\
\hline & No. & $\%$ & No. & $\%$ & No. & $\%$ & No. & $\%$ & No. & $\%$ & No. & $\%$ \\
\hline 1.Cardiovasular & & & & & & & & & & & & \\
\hline - Hypotension & - & - & - & - & - & - & - & - & - & - & - & - \\
\hline - Hypertension & - & - & - & - & 3 & 10.0 & 1 & 3.3 & 1 & 3.3 & - & - \\
\hline - Bradycardia & - & - & - & - & - & - & - & - & - & - & - & - \\
\hline - Tachycardia & 3 & 10.0 & 1 & 3.3 & - & - & - & - & - & - & - & - \\
\hline - Myocardial infarction & - & - & - & - & - & - & - & - & 1 & 3.3 & - & - \\
\hline - Arrhythmias & - & - & - & - & - & - & - & - & - & - & - & - \\
\hline - Heart failure & - & - & - & - & - & - & - & - & - & - & - & - \\
\hline 2. Gastrointestinal & & & & & & & & & & & & \\
\hline $\begin{array}{l}\text { - Nausea } \\
\text { - Vomiting }\end{array}$ & $\begin{array}{l}3 \\
3\end{array}$ & $\begin{array}{l}10.0 \\
10.0\end{array}$ & $\begin{array}{l}2 \\
-\end{array}$ & $\begin{array}{c}6.7 \\
-\end{array}$ & $\begin{array}{l}6 \\
-\end{array}$ & 20 & 2 & $\begin{array}{c}6.7 \\
-\end{array}$ & 7 & 23.3 & 2 & $\begin{array}{r}6.7 \\
-\end{array}$ \\
\hline - Gastric irritation & - & - & - & - & 12 & 40 & 3 & 10 & 9 & 30 & 1 & 3.3 \\
\hline - Constipation & 2 & 6.7 & 1 & 3.3 & 5 & 16.7 & 1 & 3.3 & 6 & 20.0 & - & - \\
\hline 3. Metabolic & & & & & & & & & & & & \\
\hline - Hyperglycemia & - & - & - & - & - & - & - & - & 1 & 3.3 & - & - \\
\hline - Hypoglycemia & - & - & - & - & - & - & - & - & - & - & - & - \\
\hline - Hyponatremia & - & - & - & - & - & - & - & - & - & - & - & - \\
\hline $\begin{array}{l}\text { 4. Wound } \\
\text { complications }\end{array}$ & & & & & & & & & & & & \\
\hline - Wound infection & 1 & 3.3 & - & - & 2 & 6.7 & - & - & - & - & - & - \\
\hline $\begin{array}{l}\text {-Impaired wound } \\
\text { healing }\end{array}$ & 1 & 3.3 & - & - & - & - & - & - & - & - & - & - \\
\hline p- value & & $\mathrm{P}=0$. & $2 n . s$ & & & & & & & & & \\
\hline
\end{tabular}


Table (15): Distribution of patients (study and control groups) as regard neurosurgical postoperative complications at follow up.

\begin{tabular}{|c|c|c|c|c|c|c|c|c|c|c|c|c|}
\hline \multirow{3}{*}{$\begin{array}{l}\text { Neurosurgical } \\
\text { postoperative complications } \\
\text { (More than one) }\end{array}$} & \multicolumn{4}{|c|}{ Before discharge } & \multicolumn{4}{|c|}{ After 3 months } & \multicolumn{4}{|c|}{ After 6 months } \\
\hline & \multicolumn{2}{|c|}{$\begin{array}{c}\text { Control } \\
(\mathbf{n}=\mathbf{3 0})\end{array}$} & \multicolumn{2}{|c|}{$\begin{array}{l}\text { Study } \\
(\mathbf{n}=\mathbf{3 0})\end{array}$} & \multicolumn{2}{|c|}{$\begin{array}{c}\text { Control } \\
(\mathbf{n}=30)\end{array}$} & \multicolumn{2}{|c|}{$\begin{array}{l}\text { Study } \\
(\mathbf{n}=\mathbf{3 0})\end{array}$} & \multicolumn{2}{|c|}{$\begin{array}{c}\text { Control } \\
(\mathbf{n}=\mathbf{3 0})\end{array}$} & \multicolumn{2}{|c|}{$\begin{array}{l}\text { Study } \\
(\mathbf{n}=\mathbf{3 0})\end{array}$} \\
\hline & No. & $\%$ & No. & $\%$ & No. & $\%$ & No. & $\%$ & No. & $\%$ & No. & $\%$ \\
\hline 1. Behavioral changes & 4 & 14.3 & 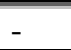 & - & 3 & 10.0 & - & - & 3 & 10.0 & - & - \\
\hline $\begin{array}{l}\text { 2. Postoperative subdural } \\
\text { hematoma }\end{array}$ & - & - & - & - & - & - & - & - & 1 & 3.3 & - & - \\
\hline 3. Seizures & 3 & 10.0 & 1 & 3.3 & 5 & 16.7 & 2 & 6.7 & 8 & 26.7 & - & - \\
\hline $\begin{array}{l}\text { 4. Residual neurological } \\
\text { problems }\end{array}$ & 4 & 14.3 & 4 & 14.3 & 6 & 20 & 4 & 14.3 & 7 & 23.3 & 3 & 10.0 \\
\hline 5. Visual disturbances & - & - & - & - & 2 & 6.7 & 2 & 6.7 & 6 & 20 & 2 & 6.7 \\
\hline 6. Brain abscess & - & - & - & - & - & - & - & - & 1 & 3.3 & - & - \\
\hline 7. Insomnia & 4 & 14.3 & 2 & 6.7 & 3 & 10 & 1 & 3.3 & 4 & 14.3 & 1 & 3.3 \\
\hline p- value & & $\mathrm{P}=0$. & $\overline{85 n . s}$ & & & $\overline{\mathrm{P}=0.2}$ & $\overline{37 n . s}$ & & & & & \\
\hline
\end{tabular}

Table (16): Comparison between assessment and follow up total score of patients' knowledge (study and control groups).

\begin{tabular}{|c|c|c|c|c|c|c|c|}
\hline \multirow{2}{*}{ Patients` knowledge } & \multicolumn{3}{|c|}{ Control $(\mathbf{n}=30)$} & \multicolumn{3}{|c|}{ Study $(\mathrm{n}=\mathbf{3 0})$} & \multirow[t]{2}{*}{ P-value } \\
\hline & No. & $\%$ & $\mathrm{X} \pm \mathrm{SD}$ & No. & $\%$ & $\mathrm{X} \pm \mathrm{SD}$ & \\
\hline $\begin{array}{l}\text { At assessment } \\
\text { Satisfactory } \\
\text { Unsatisfactory }\end{array}$ & $\begin{array}{c}- \\
30\end{array}$ & $\begin{array}{c}- \\
100\end{array}$ & $13.73 \pm 5.97$ & - & $\begin{array}{c}- \\
100\end{array}$ & $12.26 \pm 4.50$ & $\mathrm{P}=0.273 \mathrm{n} . \mathrm{s}$ \\
\hline $\begin{array}{l}\text { Before discharge } \\
\text { Satisfactory } \\
\text { Unsatisfactory }\end{array}$ & $\begin{array}{c}- \\
30\end{array}$ & $\begin{array}{c}- \\
100\end{array}$ & $14.46 \pm 4.84$ & $\begin{array}{c}30 \\
-\end{array}$ & $\begin{array}{c}100 \\
-\end{array}$ & $47.23 \pm 3.16$ & $\mathrm{P}<0.0001 * * *$ \\
\hline $\begin{array}{l}\text { After } 3 \text { months } \\
\text { Satisfactory } \\
\text { Unsatisfactory }\end{array}$ & $\begin{array}{c}- \\
30\end{array}$ & $\begin{array}{c}- \\
100\end{array}$ & $17.80 \pm 5.12$ & $\begin{array}{c}28 \\
2\end{array}$ & $\begin{array}{c}93.3 \\
6.7\end{array}$ & $45.73 \pm 4.57$ & $\mathrm{P}<0.0001 * * *$ \\
\hline $\begin{array}{l}\text { After } 6 \text { months } \\
\text { Satisfactory } \\
\text { Unsatisfactory }\end{array}$ & - & $\begin{array}{c}- \\
100\end{array}$ & $19.49 \pm 5.63$ & $\begin{array}{c}27 \\
3\end{array}$ & $\begin{array}{l}90.0 \\
10.0\end{array}$ & $43.36 \pm 4.83$ & $\mathrm{P}<0.0001 * * *$ \\
\hline
\end{tabular}

N.B. total score of knowledge is 50.

Table (17): Comparison between short form-36 questionnaire ( 8 scales plus health comparison question) of studied patients (study and control groups) after 6 months.

\begin{tabular}{|l|c|c|c|}
\hline \multicolumn{1}{|c|}{ Scales } & $\begin{array}{c}\text { Control }(\mathbf{n}=\mathbf{3 0}) \\
\mathbf{X} \pm \text { SD }\end{array}$ & $\begin{array}{c}\text { Study }(\mathbf{n}=\mathbf{3 0}) \\
\mathbf{X} \pm \text { SD }\end{array}$ & P-value \\
\hline 1. Physical functioning & $72.83 \pm 8.51$ & $89.83 \pm 10.54$ & $\mathrm{P}<0.02^{*}$ \\
\hline 2. Role limitation due to physical problems & $55.00 \pm 3.8$ & $89.67 \pm 11.4$ & $\mathrm{P}<0.001^{* *}$ \\
\hline 3. Role limitation due to emotional problems & $31.85 \pm 6.41$ & $97.0 \pm 3.00$ & $\mathrm{P}<0.0001^{* * *}$ \\
\hline 4. Vitality; energy /fatigue & $32.70 \pm 9.72$ & $53.16 \pm 11.48$ & $\mathrm{P}<0.04^{*}$ \\
\hline 5. Emotional well being & $48.28 \pm 15.58$ & $66.26 \pm 9.71$ & $\mathrm{P}<0.03^{*}$ \\
\hline 6. Social functioning & $57.41 \pm 11.5$ & $85.0 \pm 14.8$ & $\mathrm{P}<0.04^{*}$ \\
\hline 7. Pain & $60.50 \pm 3.02$ & $88.45 \pm 10.25$ & $\mathrm{P}<0.0001^{* * *}$ \\
\hline 8. General health perception & $39.50 \pm 12.86$ & $73.67 \pm 18.70$ & $\mathrm{P}<0.001^{* *}$ \\
\hline
\end{tabular}


Table (18): Comparison between health change (Health promotion) of studied patients (study and control groups) after 6 months.

\begin{tabular}{|l|c|c|c|c|c|}
\hline \multirow{2}{*}{ Health change (Health promotion) } & \multicolumn{2}{|c|}{ Control(n=30) } & \multicolumn{2}{c|}{ Study (n=30) } & \multirow{2}{*}{ P-value } \\
\cline { 2 - 5 } & No. & \% & No. & \% & \multirow{2}{*}{ P $<0.0001 * * *$} \\
\hline Much better now than 6 months ago (100) & 6 & 20.0 & 15 & 50.0 & \\
\hline Somewhat better now than 6 months ago (75) & 7 & 23.3 & 12 & 40.0 & \\
\hline About the same as 6 months ago (50) & 7 & 23.3 & 2 & 6.67 & \\
\hline Somewhat worse than 6 months ago (25) & 6 & 20.0 & 1 & 3.33 & \\
\hline Much worse than 6 months ago (0) & 4 & 13.3 & 0 & 0.0 & \\
\hline
\end{tabular}

Table (1): This table illustrates that, more than half of patients were males in both control $(60.0 \%)$ and study $(56.7 \%)$ groups. More than half of patients in control $(63.3 \%)$ and study $(56.7 \%)$ groups were having an age ranged from $(35<50)$ years with a mean value of $(43.27 \pm 6.38)$ and $(41.25 \pm 5.83)$. Regarding the patients` marital status; the majority of patients were married which include $(63.3 \%)$ in control and $(86.7 \%)$ in study groups. Regarding education; $(20.0 \%)$ in control and $(26.7 \%)$ in study groups were secondary school education and $(56.7 \%)$ in control and (46.7\%) in study groups were illiterate. Regarding occupation; (46.7\%) of patients in control and (36.7\%) in study groups were manual work.

Table (2): Illustrates that, more than half of patients in control $(60.0 \%)$ and less than half of patients in study $(43.33 \%)$ groups performed craniotomy.

Table (3): GCS of all patients in both study and control groups presented with a mean value of $(15.00 \pm 15.00)$.

Table (4): There was non significant difference between study and control groups as regard cranial nerves assessment.

Table (5): There was non significant difference between study and control groups as regard sensory function assessment.

Table (6): There was non significant difference between study and control groups as regard sphincter control assessment.

Table (7): There was non significant difference between study and control groups as regard motor function assessment.

Table (8): Shows the difference between patients` vital signs recording chart for both study and control group. There were significant differences during the 3rd day postoperative in vital signs including temperature, pulse, respiration and blood pressure [systolic/diastolic] $(\mathrm{P}<0.01, \mathrm{P}<0.01, \mathrm{P}<0.04,[\mathrm{P}<0.04$, $\mathrm{P}<0.02])$ respectively. A mean value for control group (37.85 $\pm 0.39,77.00 \pm 11.84,77.00 \pm 11.84$, $[116.67 \pm 5.77,78.73 \pm 4.86])$ respectively. A mean value for study group $(36.92 \pm 0.15,79.80 \pm 4.55$, $18.20 \pm 1.39, \quad[115.57 \pm 19.66, \quad 77.63 \pm 3.87])$ respectively.
Table (9): shows that, the majority of patients in both control $(93.3 \%)$ and study $(90.0 \%)$ groups were having anxiety and fear.

Table (10): There was non significant difference between study and control groups as regard laboratory investigation.

Table (11): There was non significant difference between study and control groups as regard diagnostic procedure.

Table (12): There was non significant difference $(\mathrm{P}=0.273)$ between study and control groups as regard systemic complications at assessment.

Table (13): There was non significant difference $(\mathrm{P}=0$. 338) between study and control groups as regard neurosurgical complications at assessment.

Table (14): There was non significant difference $(\mathrm{P}=0.252)$ between study and control groups as regard systemic complications before discharge. After 3 and 6 months of follow up there was significant difference regarding systemic complications ( $\mathrm{P}<0.03, \mathrm{P}<0.04)$ respectively.

Table (15): There was non significant difference between study and control groups as regard neurosurgical complications before discharge and after 3 months of follow up( $\mathrm{P}=0.485, \mathrm{P}=0.237)$. There was significant difference $(\mathrm{P}<0.01)$ after 6 months of follow up regarding neurosurgical complications.

Table (16): This table shows that, all patients (study and control groups) had unsatisfactory level of knowledge at the time of assessment and had non significant difference $(P=0.273)$. At the time of follow up, there was high significant difference ( $P$ $<0.0001$ ) between patients knowledge (study and control groups) in relation to total knowledge score.

Table (17): This table illustrates that, there was high significant difference $(\mathrm{P}<0.0001)$ between study and control groups as regard role limitation due to emotional problems and pain. There was moderate significant difference $(\mathrm{P}<0.001)$ between study and control groups as regard role limitation due to physical health problems and general health perception. There was significant difference between study and control groups as regard physical 
functioning, vitality, emotional well being and social functioning $(\mathrm{P}<0.02, \mathrm{P}<0.04, \mathrm{P}<0.03, \mathrm{P}<0.04)$ respectively.

Table (18): Illustrates that, there was high significant difference $(\mathrm{P}<0.0001)$ between study and control groups as regard health change. The majority of patients $(90 \%)$ in study group were having health improvement while less than half of patients in control group were having health improvement.

\section{Discussion}

The present study aimed to:

1.Determine the physical, social and emotional problems of patients after intracranial surgery.

2. Identify the needs of patients after intracranial surgery.

3. Develop and implement the nursing educational program based on patient's needs.

One of the most critical surgeries performed on the human body is intracranial surgery. The entire treatment plan affects the patient physically, mentally and emotionally. To accomplish quality care and best possible outcome after intracranial surgery; nurses should be knowledgeable of the type of surgery planned, its course and possible complications (Guilabert, 2014).

The results of the present study showed that more than half of patients in both control and study groups were males their age ranged from $35<50$ years old and the majority of them were married and employ. In this regard this result agree with the study of (BinMadhi, 2012) entitled as "Brain tumors excision guided by neuronavigation: Practical application and results " which revealed that intracranial surgery is more common in males than females with a mean age of 47 years old.

The results in the present study revealed that patients $\mathrm{GCS}=15$ at the time of assessment after intracranial surgery indicate good prognosis for both study and control groups. Most of patients after intracranial surgery are clinically improved because pressure of the tumor, haematoma, abscess or cyst is relived.

In the same line this result agree with the study of (Abbass et al., 2007), entitled as (Glasgow coma scale on admission is correlated with postoperative Glasgow outcome scale in chronic subdural hematoma) which revealed that their is positive correlation between admission GCS and GCS in chronic subdural hematoma.

Through researcher's assessment, found that blood sample may be drawn after intracranial surgery to determine the level of red blood cells, white blood cells, platelets, hemoglobin, concentration of sodium and potassium because certain changes may be occurred after intracranial surgery. Also, certain procedures may be performed to evaluate patients conditions and this is performed according to their clinical presentation.

The results in the present study showed that there were non significant differences at the time of assessment between patients and their neurological physical assessment after intracranial surgery such as cranial nerves, motor function, sensory function, sphincter control. There were non significant differences between patients and their laboratory investigation and diagnostic procedures after intracranial surgery.

In the same line (Brem, 2014) stated that frequent neurological checks will be performed by the nursing and medical staff to test the brain function and to make sure the body systems are functioning properly after surgery. Patients will be asked to follow a variety of basic commands, such as moving arms and legs, to assess brain function. Pupils will be checked with a flash light, and patients will be asked questions to assess their orientation (such as name, date, and place). The strength of arms and legs will also be tested.

These results supported by (Godoy, 2013) who reported that laboratory investigations and skull computed tomography should be obtained to establish a diagnosis of the complications and any postoperative neurological deteriorations detected during neurological examination and to plan new intervention, if necessary. (Edlinger et al., 2012) stated that to manage a postoperative neurosurgical patient the care provider requires knowledge of how the central nervous system reacts to stress and anesthesia as well as the potential complications associated with each specific procedure.

As regard vital signs in the present study we found that there was significant difference of body temperature during the third postoperative day. Regarding pulse there was moderate significant difference during the second postoperative day and high significant difference during the third postoperative day. Regarding respiration there was significant difference during the third postoperative day. Regarding blood pressure there was moderate significant difference in systolic and high significant difference in diastolic blood pressure during the third postoperative day.

So, the results of the present study show no signs of increased intracranial pressure postoperative. The abnormal changes that occur in vital signs may be attributed to emotional stress and fear in control group than in study group during the second and third postoperative days and this is also the neurosurgeon opinion in the neurosurgery department. 
The presence of non significant differences in vital signs especially pulse and blood pressure during the first postoperative day can be attributed to anxiety and fear in both groups and these can produce hypertension and tachycardia as in the study sample.

However, the study of (Smith and Timby, 2013) demonstrated that the blood pressure, pulse, respiration, and temperature are closely monitored on all patients with a potential or actual neurological disorder. The temperature often needs to be monitored every hour because central nervous system disorders can affect the temperature-regulating center of the hypothalamus. A sudden increase or decrease in any of the vital signs indicates a change in the neurological status, and the physician is notified immediately.

The results of the present study showed that there were non significant differences related to systemic and neurosurgical complications at the time of assessment; before application of the nursing educational program. This may be attributed to that the majority of studied patients suffer from anxiety and fear in addition to the immediate postoperative effect of anesthesia and this is also the opinion of the neurosurgeon and anesthesiologist in the neurosurgery department.

The results of the present study showed significant difference as regard systemic complications after application of the nursing educational program at the time of follow up (after 3 and 6 months). As regard neurosurgical complications, there was significant difference after application of the nursing educational program at the time of follow up (after 6 months).

The study finding was supported by (Buttaro, 2008) who demonstrated that unmanaged stress is linked to hypertension, heart diseases and gastrointestinal problems. Also, unmanaged stress is linked to some emotional health disorders. An elevated level of stress increases the frequency of abnormal behaviours.

(Silver et al., 2011) said that patients who have a successful outcome after intracranial operation may still experience residual neuropsychological symptoms that may be not noticed by the examiner but devastating to the patient. Over the years, many patients have expressed the opinion that some discussion of such possible sequelae before and after surgery would have been helpful to them. Neurosurgeons should routinely discuss possible neurological sequelae with patients before and after brain surgery. Providing patients with a simple brochure describing possible temporary or long-term neuropsychological consequences is suggested. Such information must be shared in a manner that does not frighten or upset the patient but provides true informed consent.
In the present study, all patients in both study and control groups before application of nursing educational program for patients after intracranial surgery had unsatisfactory level of knowledge regarding intracranial surgery.

This could be explained by the fact that, patients didn't receive enough information from health care team. Some patients were lacking interest to know any information while others were interested to know but they didn't find the person who had enough time to provide them with enough information. In the same line this result agree with (Desoky, 2014) study who said that "Impact of a designed nursing teaching protocol on quality of life of patients with chronic lower limb ischemia at Assiut University Hospital" which revealed that all patients in both study and control groups had unsatisfactory level of knowledge before application of a designed nursing teaching protocol.

After application of the nursing educational program for patients after intracranial surgery (before discharge, after 3 months and after 6 months), patients` knowledge score levels regarding intracranial surgery were highly significantly improved for study group patients. This improvement emphasis the fact that, most patients have a strong desire to learn more knowledge about their conditions and show the effect of the program.

This result is in the same line with the study of (Ali, 2004) entitled as "Impact of a designed nursing intervention protocol on performing self-care activities among rheumatoid arthritic women" which revealed that all studied sample had unsatisfactory level of knowledge about their disease at initial assessment, however, after application of a designed nursing intervention protocol, founded that, there was a significant increase in knowledge of study group patients regarding disease.

The results of the present study showed positive effect for the nursing educational program on patients` recovery and health for study group patients. Health promotion in this study was evaluated by assessing postoperative complications at the time of follow up and by using SF-36 questionnaire. The SF36 self-evaluated health transition items (five response categories ranging from "much better" to "much worse") has been shown to be useful in estimating average changes in health status. It is individualized questionnaire; measured according to patient's response and patient's response differ from one patient to another.

These results were supported by (Piper and Stewart, 2009) who revealed that effective health educational program will result in changes that demonstrate increased knowledge about specific medical and health-related issues for a prolonged period of time. 
Effective health education will yield both short-term and long-term changes in behavior that reduce risky behavior and/or improve quality-of-life. These changes in behavior can be recorded through evaluator observations and learner feedback, or through more formal means such as questionnaires. Before discharge there was improvement in the scales concerned with role limitations due to emotional problems, social functioning and general health. After 3 months there was improvement in the scale concerned with role limitations due to emotional problems, vitality, mental health, social functioning, and general health. After 6 months there was improvement in all 8 scales plus health comparison question (health change).

Regarding health comparison question, this question was asked to the patients at the last time of follow up (after 6 months) to show the effect of the nursing educational program. The majority of patients $(90 \%)$ in study group were having health improvement while less than half of patients $(43 \%)$ in control group were having health improvement.

In this regard this result agree with the study of (BinMadhi, 2012) entitled as "Brain tumors excision guided by neuronavigation: Practical application and results " which revealed $85 \%$ of postoperative patients showed an improvement of their neurological status.

However, (Krug, 2008) who conducted study entitled as "Functional outcome and self-perceived overall health status following surgery to remove primary brain tumor" which revealed that postoperative complications may delay improvement, though at three months, these complications and their effect on function should have been overcome. Functional recovery following surgery for brain tumor was not significant overall. When examined individually, a majority of subjects demonstrated improvement of a clinically relevant nature.

\section{Conclusions}

Based on the result of the present study, it can be concluded that:

- Patients after intracranial surgery; burr holes, craniotomy and craniectomy are at high risk for systemic and/or neurosurgical postoperative complications which had a bad effect on patients` health.

- Before application of the nursing educational program all patients in both study and control groups had unsatisfactory level of knowledge.

- The 1st hypothesis was supported as patients knowledge score levels were highly significantly improved for study group patients after application of nursing educational program. Control group patients who did not receive the nursing educational program had unsatisfactory level of knowledge at the time of follow up.

- The 2nd hypothesis was supported as a highly significant difference between study and control groups in relation to health improvement. The majority of patients in study group were having health improvement while less than half of patients in control group were having health improvement after application of nursing educational program.

- The 3rd hypothesis was supported as the study group patients suffered from less symptoms or complications (systemic or neurosurgical); physical, social or emotional problems than control group patients during follow up periods.

\section{Recommendations}

Based on results of the present study the following can be recommended:

\section{For patients}

Patients are to be provided with sufficient information to remind them with specific instructions regarding treatment, how to cope with postoperative periods; wound care, activity, rest, diet, stress and maintain effective communication to avoid certain postoperative complications that may develop and when they must notify physician.

\section{For nurses}

Nurses should be aware by instructions that given to patients before discharge and inform patients about them to improve their health.

\section{For administration}

Establishment of health care educational center in the neurosurgery department to educate patients about necessary instructions regarding their conditions using booklet and illustrated pamphlets for each patient especially those who cannot read and write.

\section{For research}

Similar studies should be replicated on longitudinal bases till one year as a minimum time period for follow up.

\section{References}

1. Abbass, A., Abouzari, M., and Rashidi, A., (2007): Glasgow Coma Scale on admission is correlated with postoperative Glasgow Outcome Scale in chronic subdural hematoma, Journal of Clinical Neuroscience,14(12), pp.1240-1241

2. Ali, J.., (2004): Impact of a designed nursing intervention protocol on performing self-care activities among rheumatoid arthritic women, Unpublished doctor thesis, Medical-Surgical Nursing, Faculty of nursing, Assiut University, p.104 
3. Bin-Madhi, M., (2012): Brain tumors excision guided by neuronavigation: Practical application and results, Unpublished doctor thesis, Neurosurgery, Faculty of medicine, Assiut University, pp. 89, 92, 95, 99

4. Brem, H., (2014): Craniotomy, The Johns Hopkins University, The Johns Hopkins Hospital, and Johns Hopkins Health System. http://www.hopkinsmedicine.org/healthlibrary/te st_procedures/neurological/craniotomy_92,p087 67/, Published online 18-1-2014

5. Buttaro, (2008): Self-esteem disturbance clinical validation study, In Mclane AM, Classification nursing diagnosis, Proceedings of the seventh C. ence. St. Louis, Volume 15, P. 8690.

6. Desoky, A., (2014): Impact of a designed nursing teaching protocol on quality of life of patients with chronic lower limb ischemia at Assiut University Hospital, Unpublished doctoral thesis, Medical-Surgical Nursing, Faculty of nursing, Assiut University, pp. 62-64, 87

7. Edlinger, M., Strohmaier, S., Jonsson, H., (2012): Blood pressure and other metabolic syndrome factors and risk of brain tumour in the large population-based Me-Can cohort study, Journal of Hypertension, 30(2), pp.290-296

8. Godoy, A., (2013): Intensive Care in Neurology and Neurosurgery,1st ed., Lippincott Williams and Wilkins, p.1514

9. Guilabert, H., (2014): The postoperative cranium, normal findings and complications, European Society of Radiology, p.2, www.myESR.org, Published online 21-7-2014

10. Haddad, S., Spir, A., Craft, S., and Hart, S., (2013): Benefits of patient education, Patient Education Institute, http://ww2.patienteducation.com/main.asp?p=aboutus\&s=bope \&fs $=$ aboutus $\&$ mode $=$ FULL , Published online 3-122013

11. Heisler, J., (2014): Craniotomy versus craniectomy, about health, http://surgery.about.com/od/proceduresaz/qt/Cra niotomyCraniectomy.htm, Published online 15$12-2014$

12. Hospital record, (2013-2014): Neurosurgery department, Assiut University Hospital.

13. Krug, J., (2008): Functional outcome and selfperceived overall health status following surgery to remove primary brain tumor, Unpublished master thesis, Faculty of the Graduate School, University of Missouri

14. McGlinsey, A., and Kirk, A., (2014): Neurological assessment, Early identification of neurological deterioration is vital to preventing secondary brain injury,
http://nursing.advanceweb.com/ContinuingEducation/CE-Articles/neurologicalassessment.aspx, Published online 16-1-2014

15. Piper, S., and Stewart, P., (2009): Health Promotion for Nurses: Theory and practice, United Kingdom: Taylor and Francis, British Journal of General Practice, 25 (6), pp. 294-305.

16. Silver, M., McAllister, W., and Yudofsky, C. (2011): Text book of traumatic brain injury, ${ }^{2 n d}$ ed., American psychiatric association, pp. 427436

17. Smith, E., and Timby, K., (2013): Introductory Medical-Surgical Nursing, $11^{\text {th }}$ ed., Lippincott Williams and Wilkins, pp. 560-570

18. Suzanne, C., Smeltzer, O., Bare, G., Hinkle, L., and Cheever,H.K., (2010): Brunner and Suddarth's Text book of Medical-Surgical Nursing, Chapter 65, $12^{\text {th }}$ ed., Wolters Kluwer health/ Lippincott Williams and Wilkins, pp. 1983, 1984

19. Teasdale, G., and Jennett, B., (1974): Assessment of coma and impaired consciousness, practical scale, Glasgow Coma Scale, University of Glasgow.

20. Ware, J., and Sherbourne, C., (1992): The Medical Outcomes Study 36-item short-form health survey, RAND corporation, Medical care, version 1.0, 30(6), pp.473-483.

21. Wilson, M., Wise, D., Davies, G., and Lockey, D., (2013): Emergency burr holes: How to do it, Scandinavian journal of trauma, resuscitation and emergency medicine, U.S. national library of medicine, national institute of health, Volume 20 , pp. $20-24$

22. World Health Organization, (2012): Collaborating Center for Evidence-Based Health Promotion in Hospitals and Health Services, Promoting hospitals-network-hph.htm, Published online 17-2-2012.

23. Yarbro, H., Wujcik, D., and Gobel, H., (2011): Cancer Nursing: Principles and practice, Central nervous system cancers, Chapter $49,7^{\text {th }}$ ed., Jones and Barlett Publisher, LIC., p. 1169 